\title{
Wind-load, pressure and velocity measurements: post processing and discussion
}

\author{
Md. Mahbub Alam ${ }^{1, a}$ \\ ${ }^{1}$ Institute for Turbulence-Noise-Vibration Interaction and Control, Harbin Institute of Technology, Shenzhen Graduate \\ School, Shenzhen, China
}

\begin{abstract}
Because of the practical importance of bluff bodies subjected fluid flow, fluid dynamics around two-dimensional bluff bodies, especially circular cylinders, has been studied in light of three major parameters, namely flow-induced force, surface pressure and wake. Though all the three are interrelated, the first two are the integrated behavior of flow velocity around the cylinder. Thus measurements of flowinduced forces, surface pressures and velocity fields are very important in the engineering discipline. This paper discusses force and pressure measurement results for two circular cylinders at gap-spacing ratio $T / D$ varied from 0.1 to 5 and the attack angle $\alpha$ from $0^{\circ}$ to $180^{\circ}$ where $T$ is the gap width between the cylinders and $D$ is the diameter of a cylinder. Post processing of pressure and force signals is also elaborated.
\end{abstract}

\section{Introduction}

Body and flow are two different perspectives to examine the aerodynamic force on the body. According to Newton's third law, the force on the body due to the fluid flow is opposite to the force imposed by the body on the fluid flow. Estimation of aerodynamic forces on the body perspective includes the integration of the normal and tangential forces acting on the body. On the other hand, that in flow perspective is done using the momentum conservation equation for a control volume enclosing the body. Surface pressure is measured with a pressure tap connected to manometer. When a bluff body is subjected to a flow, vortex shedding from the body occurs in an alternating fashion, resulting in fluctuating forces acting on the body. The fluctuating forces in some cases generate vortex excited vibration or galloping vibration, causing a catastrophic failure of the body. Therefore both steady and fluctuating (unsteady) force components are equally important to the designers. In both force and pressure measurement techniques, measurements of unsteady components using classical techniques are still challenging. Being a representative, a time-history of single point velocity in the wake can reflect the major behavior of the wake. For example, Strouhal number $(S t)$ estimated from the a single point velocity signal gives us

\footnotetext{
${ }^{\mathrm{a}}$ Corresponding author: alamm28@yahoo.com
}

an image of the wake structure as well as forces acting on the body.

Here measurements of forces, pressure and singlepoint-velocity measurements on two circular cylinders in various arrangements are the main focus. Two fluiddynamically interfering cylinders may be considered as the basic element of multiple structures and the knowledge of this flow is insightful for understanding the flow around more structures. As such, the flow around two cylinders has received a great deal of attention in the literature. There is no doubt that flow physics around two cylinders is much more complex and complicated than that around a single cylinder, because of interference between the cylinders, between the wakes, among four shear layers, etc. Thus sophisticated processing of force, pressure and velocity signals are required to extract the complicated physics.

Sumner et al. [1] conducted flow visualization and particle image velocimetry (PIV) measurements for $T / D$ $=1.0 \sim 5.0, \alpha=0^{\circ} \sim 90^{\circ}$ and Reynolds number $\operatorname{Re}=850$ - $1900\left(R e=U_{\infty} D / v\right.$, where $U_{\infty}$ is the free-stream velocity and $v$ is the kinematic viscosity), and divided the $T / D-\alpha$ plane into three: (1) the single body flow regime, $T / D=$ $1.0 \sim 1.125$ and $\alpha=0^{\circ} \sim 90^{\circ}$, where two cylinders act like an isolated body with a single vortex-shedding frequency; (2) the small incidence angle regime, $T / D>$ 1.125 and $\alpha=0^{\circ} \sim 20^{\circ}$, where shear layer reattachment 
or the impingement of vortices onto the downstream cylinder takes place; (3) the large incidence angle regime, $T / D>1.125, \alpha=20^{\circ} \sim 90^{\circ}$, where vortex pairing, splitting, enveloping and synchronizing occur. $\mathrm{Gu}$ and Sun [2] measured the time-averaged pressure on two cylinders $\left(T / D=0.1 \sim 2.5\right.$ and $\left.\alpha=0^{\circ} \sim 90^{\circ}\right)$, and observed three distinct pressure distributions, viz. Patterns $\mathrm{I}_{\mathrm{B}}, \mathrm{II}_{\mathrm{B}}, \mathrm{III}_{\mathrm{B}}$, on the downstream cylinder, which occurred over $\alpha=0^{\circ} \sim 9.65^{\circ}, 9.7^{\circ} \sim 15^{\circ}$, and $16^{\circ} \sim 90^{\circ}$, respectively, at $T / D=0.7$.

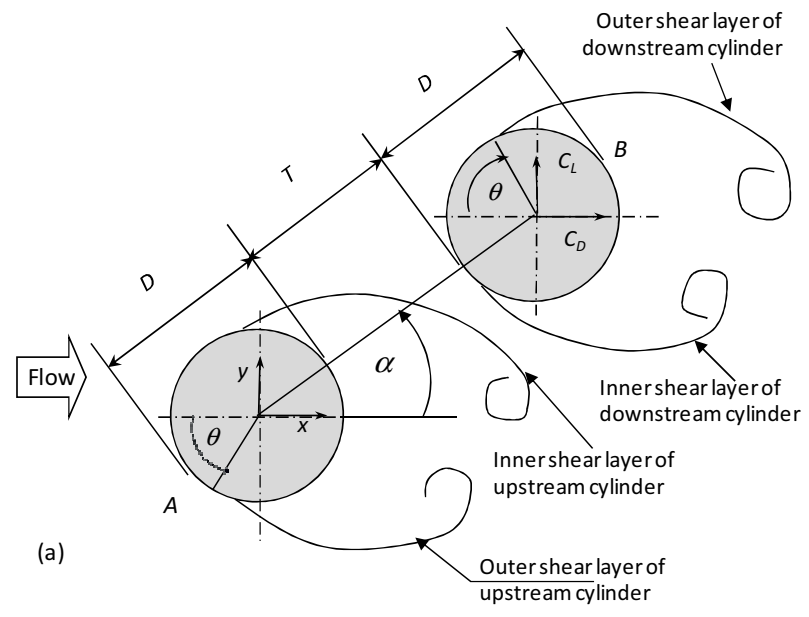

(b)

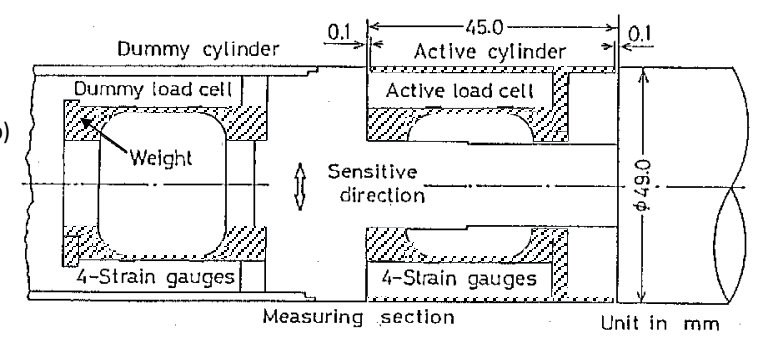

Figure 1. (a) Arrangement of cylinders and definitions of symbols. (b) Installation of load cells inside cylinder B.

Time-mean drag and lift forces acting on two staggered cylinders have been examined in the literature (e.g., [3-4]), with most of the emphasis being on the downstream cylinder. Only a few studies have reported force measurements for the upstream cylinder [2, 5, 6]. Furthermore, fluctuating force measurements in the literature are very scant, though the fluctuating lift and drag forces acting on structures are major causes of the fatigue failure of the structures and are used for predicting flow-induced responses. The present study indemnifies all possible quantities including mean and fluctuating forces, $S t$ and pressure. Flow visualization and surface oil-flow techniques are also employed to get insight into the physical flow around the cylinders and to get a better understanding of the mean and fluctuating forces and $S t$.

\section{Experimental details}

Experiments were conducted in a low-speed, closedcircuit wind tunnel with a test section of $1.20 \mathrm{~m}$ in height, $0.30 \mathrm{~m}$ in width, and $2.2 \mathrm{~m}$ in length. Figure $1(\mathrm{a})$ is a schematic diagram showing the arrangement of two cylinders, definition of symbols and coordinate systems. The diameter $D$ of each cylinder was $49 \mathrm{~mm}$. The stagger angle $\alpha$ is defined as the angle between the free-stream flow and the line connecting the centers of the two cylinders. $T$ is the gap width between the cylinders. The position of a point on the surface of a cylinder is defined by the azimuth angle $\theta$, measured from the direction of free-stream flow. $\theta$ is considered $0^{\circ} \sim 180^{\circ}$ for the freestream side surfaces and $180^{\circ} \sim 360^{\circ}$ for the gap-flow side surfaces of the cylinders. The cylinder $A$ is tentatively assumed to be fixed, and traversing of the cylinder $B$ can be done with variations of the two parameters $T / D$ and $\alpha$. Fluid forces, St, and cylindersurface pressure measurements and surface oil-flow visualization were conducted at a $R e$ of $5.52 \times 10^{4}$ based on the diameter of a cylinder. The turbulent intensity was $0.5 \%$. Experiments were performed for $\alpha=0^{\circ}, 10^{\circ}, 25^{\circ}$, $45^{\circ}, 60^{\circ}, 75^{\circ}, 90^{\circ}, 105^{\circ}, 120^{\circ}, 135^{\circ}, 155^{\circ}, 170^{\circ}$, and $180^{\circ}$, for the spacing ratio of $T / D=0.1 \sim 5$. Very fine-tuning of $T / D$ was adopted with $T / D=0.1,0.2,0.3,0.5,0.6,0.7$, $0.8,0.9,1.1,1.2,1.5,1.8,2.1,2.4,2.7,3.0,3.5,4.0,4.5$ and 5.0.

\section{Load cell measurements: aerodynamic forces}

In order to measure forces successfully, particularly fluctuating component, the natural frequency of the cylinder system must be higher than four times the vortex shedding frequency [7]. If a commercial load cell is used to measure forces on the cylinder, the natural frequency can be achieved between 20 to $70 \mathrm{~Hz}$, lower than the vortex shedding frequency. Consequently the performance of the load cell deteriorates to measure fluctuating forces. A special load cell of high sensitivity and high natural frequency was therefore built with eight semi-conductor strain gages. The cylinder to be measured was built in with an active ('live') section of a spanwise $45 \mathrm{~mm}(0.92 D)$ length and two dummy sections (figure 1b). This size was determined taking into account the cross-correlation length of fluctuating pressure in the spanwise direction of the cylinder. The active section, placed between the two dummy sections, corresponded to the midspan of the cylinder and was installed with a load cell that consisted of four semiconductor strain gauges. One of the dummy sections was also instrumented with another load cell of the same configuration. The load cell inside the active section measured a combination of fluid 
forces and forces due to vibration transmitted from outside through the cylinder support, whilst that inside the dummy section measured the latter forces only. Hence the fluid forces acting on the active section could be calculated by subtracting the output of the load cell inside the dummy section from that of the load cell inside the active section. The load cell had a high linearity in the load/output relation. The sensitivity of the load cells was $11.311 \mathrm{mV} / \mathrm{g}$. The natural frequency of the load cell set in the active cylinder was about $1009 \mathrm{~Hz}$, whereas the measured frequency of the fluctuating lift ranged from approximately $20-140 \mathrm{~Hz}$. Thus the natural frequency of the load cell was 7.2 - 50 times greater than the frequency of fluctuating lifts.

Time-averaged lift coefficient $\left(C_{L}\right)$, Time-averaged drag coeffiecient $\left(C_{D}\right)$, Fluctuating $(\mathrm{rms})$ drag coefficient $\left(C_{D f}\right)$ and fluctuating (rms) lift coefficient $\left(C_{L f}\right)$ are measured for the whole ranges of $\alpha$ and $T / D$. Only $C_{L}$ and $C_{L f}$ contours in a $T / D$ - $\alpha$ plane are however presented in figure 2 . In the scale bars, the color or the range marked by black ' $*$ ' indicates the value of a single isolated cylinder. The result can be described with reference to figure 1(a) in which cylinder $A$ is fixed, and traversing of cylinder $B$ is done with variations of the two parameters $T / D$ and $\alpha$, which suffice to determine the possible arrangement of the two cylinders. It may be noted that cylinder $B$ acts as the downstream cylinder for $|\alpha|<90^{\circ}$ and the upstream cylinders for $|\alpha|>90^{\circ}$, i.e. the left and right sides of a contour map show the values of coefficient of the upstream and downstream cylinders, respectively. At the peripheries of the middle and outer circles, the values of $T / D$ are 0.0 and 5.0, respectively. Upward (+ve y-direction) $C_{L}$ is considered as positive.

The $C_{L}$ in the downstream region (right half) is highly sensitive to $T / D$ and $\alpha$, however, that in the upstream region (left half) retains single-cylinder values except for $|\alpha|=135^{\circ}-180^{\circ}, T / D<0.4-1.0$, and $|\alpha|=90^{\circ}-135^{\circ}$, $T / D<1.3-0.4$. The $C_{L}$ around the cylinder for $T / D<0.5$ varies greatly with change in $\alpha$ from $0^{\circ}$ to $360^{\circ} . C_{L}$ becomes highly negative $(\approx-1.03)$ on the upstream cylinder at $\alpha=140^{\circ} \sim 170^{\circ}, T / D=0.25 \sim 0.45$ and $(\approx-$ $1.15 \sim-1.25)$ on the downstream cylinder at $\alpha=5^{\circ} \sim$ $15^{\circ}, T / D=0.4 \sim 1.7$. In these regions, highly negative $C_{L}$ is mainly due to formation of a separation bubble on the inner (gap flow side) surface or a turbulent reattachment of inner shear layer near the base of the cylinder. $C_{L}$ on both cylinders become positive in the vicinity of side-byside arrangement $\left(\alpha=90^{\circ}\right)$ at smaller spacing. These positive lift forces are mainly due to shift of stagnation point toward the gap. At the respective conjugate positions, $C_{L}$ increases to a maximum.
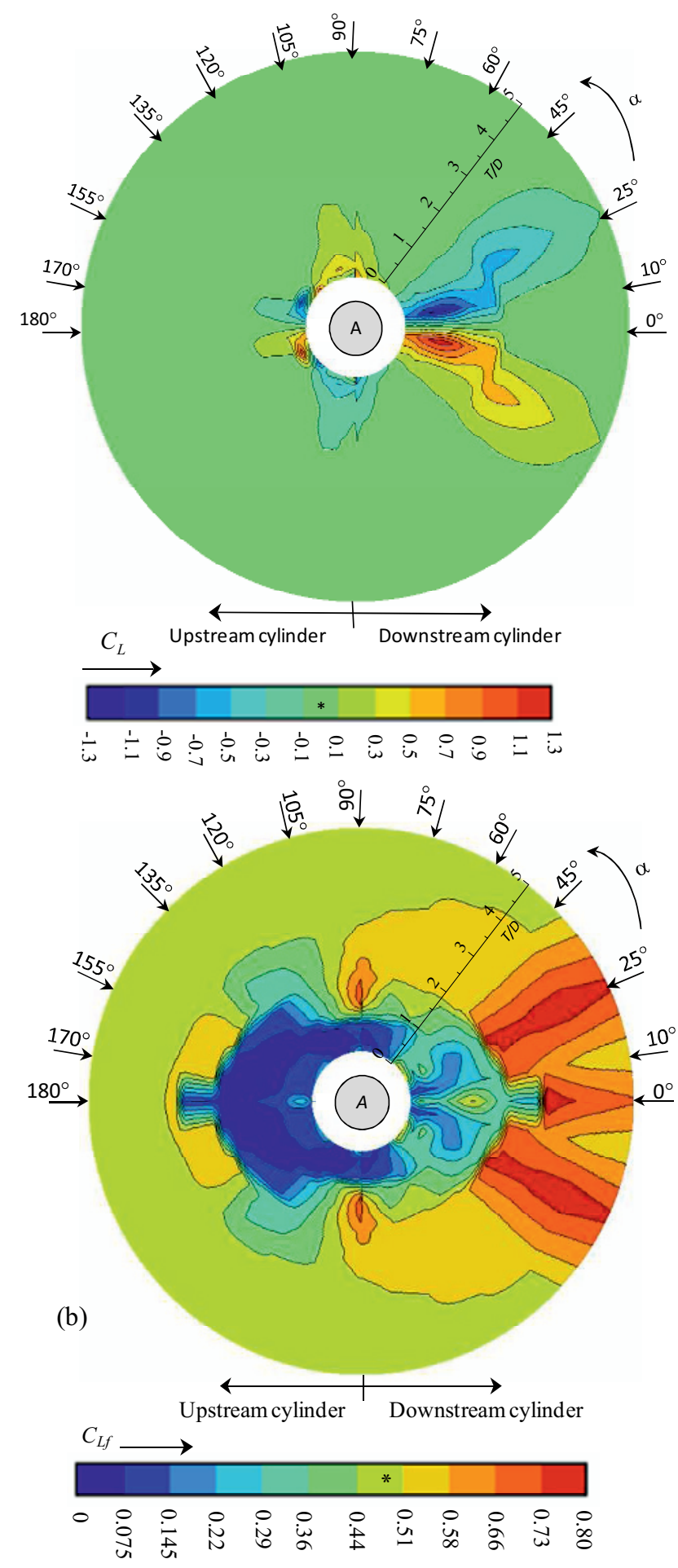

Figure 2. Time-mean lift coefficient $C_{L}$ (a) and fluctuating lift coefficient $C_{L f}$ (b) contours in $T / D-\alpha$ plane [10]. Points marked by '*' denote values of coefficients of an isolated cylinder.

The $C_{L f}$ in the upstream region retains singlecylinder values except for $T / D<2.5$. $C_{L f}$ controur displays three island-like regions, two of them are in tandem $\left(\alpha=0^{\circ}, T / D>3\right)$ and side-by-side $\left(\alpha=90^{\circ}, T / D=\right.$ 1 - 2) configurations, respectively, and the other at stagger $\left(\alpha=25^{\circ}, T / D>2.5\right)$. The global maximum value of $C_{L f}$ is $0.8,1.58$ times the single cylinder value, 
resulting from the influence the upstream cylinder vortices passing alternately underneath the downstream cylinder. The $C_{L f}$ is extremely small for $|\alpha|>60^{\circ}, T / D<$ 3.0. The observation suggests that the interference between the cylinders has not only a negative impact with increasing forces, but also a positive impact with reducing forces on the cylinder. Its impact, however, depends on $\alpha$ and $T / D$.

\section{Physical detection of bistable flow}

Pressure measurement. A semiconductor pressure transducer (Toyoda PD104K) located at the midsection of the monitored cylinder was used to measure cylinder surface pressure. Again response of the transducer should be examined before starting the experiment. The transducer responded reasonably well to the pressure fluctuations up to $500 \mathrm{~Hz}$ with a gain factor of $1 \pm 0.06$, with a negligible phase lag. This frequency was well above the frequency of vortex shedding from the cylinders. St was estimated from spectral analysis of the fluctuating pressures measured on side surfaces of the cylinders and also from the hotwire measured velocity in the wake.

Surface oil-flow. Surface oil-film technique was used to visualize the flow pattern on the cylinder surface in order to obtain information on the reattachment and separation positions of flow. The cylinders were wrapped with a black film of $0.03 \mathrm{~mm}$ in thickness, and then an even coating of a solution containing silicone oil, titanium dioxide, oleic acid and kerosene at a ratio of 45:3:2:2 in weight was painted on the surface, which was exposed to the uniform flow in the wind tunnel for at least 15 minutes. The black film was then unwrapped carefully, with the photographs of solution distribution on the film taken using a digital camera.

Conditional sampling technique. Following are the sequential steps for evaluating pressure/fluid-force coefficients separately for a case where two modes of flow pattern intermittently/randomly switch from one to the other. As an example, the signal of instantaneous lift, $C_{L i}$, of a cylinder of two side-by-side cylinders, as shown in figure 3(a), possesses two patterns of signals, with some portions having low $C_{L}$ and some having high $C_{L}$. First, as defined in equation (1), the local average $C_{L p}$ of $C_{L i}$ is calculated for the time interval corresponding to digitized $N$ data counted from a peak to the $i$ th (the next peak) data,

$$
C_{L p}(i, N)=\frac{1}{N} \sum_{j=i+1}^{i+N} C_{L i}(j) .
$$

To obtain the peaks with reasonable accuracy, highfrequency noises are eliminated from digitally stored data by using a low-pass filter, while the original data are stored. Then a probability density distribution, $P\left(C_{L p}\right)$, of the values of $C_{L p}$ is constructed to find a threshold value that divides the $C_{L}$ for the two modes as shown in figure 3(b). It is clear from the figure that the values of $C_{L p}$ are closely arranged around two values of $C_{L p}\left(C_{L p}=-0.15\right.$ and 0.55$)$. The appearance of two peaks implies that the input signal is composed of two major patterns whose individual averages are near -0.15 and 0.55 . Thus the value of $C_{L p}$ denoted by $H$ can be assumed to be a threshold value that divides the values of the two modes. By determining the threshold value $H$, the prerecorded input signal in the form of digital data into the memory is portioned out into the two modes for $C_{L p}<H$ and for $C_{L p}$ $\geq H$, respectively. Then the average and rms values of the data for one are calculated to obtain the lift coefficient, $C_{L}$, and the fluctuating lift coefficient, $C_{L f}$. Similarly, $C_{L}$ and $C_{L f}$ for the other mode are calculated. To obtained pressure distributions for the two modes separately, lift force signal of a cylinder and the pressure signal of the other cylinder were stored simultaneously, so that the lift force signal can be used as a reference signal to identify $C_{P}$ or $C_{p f}$ for the two modes.
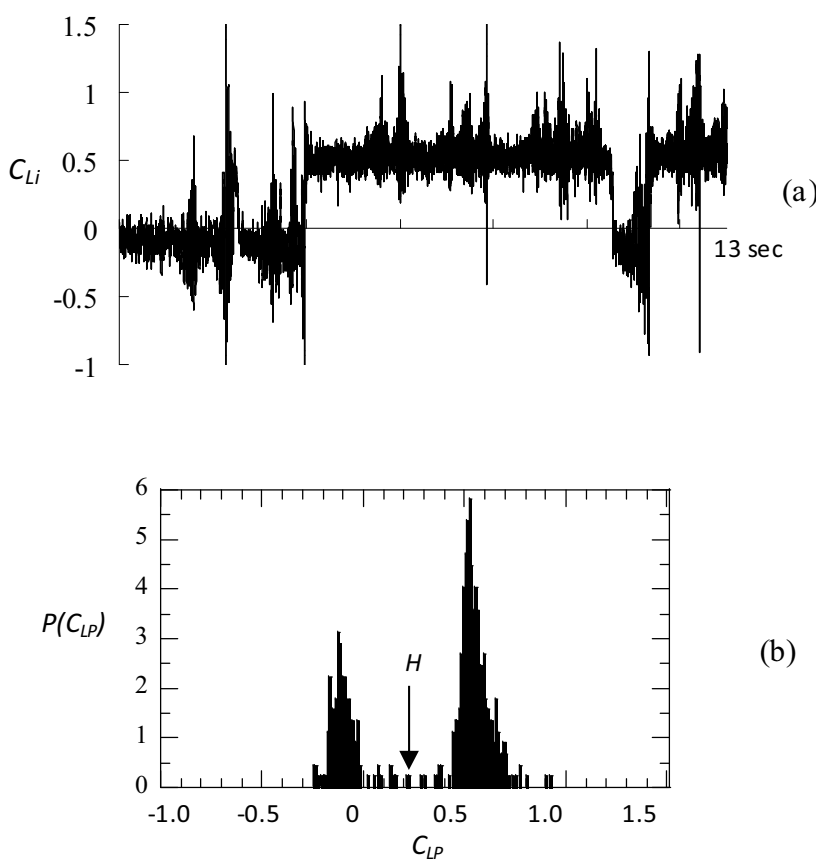

(b)

Figure 3. (a) Lift force signal of cylinder $\mathrm{B}$ at $\alpha=90^{\circ}, T / D=0.10$. (b) Probability density distribution of local average, $C_{L P}$, of signal (a). 


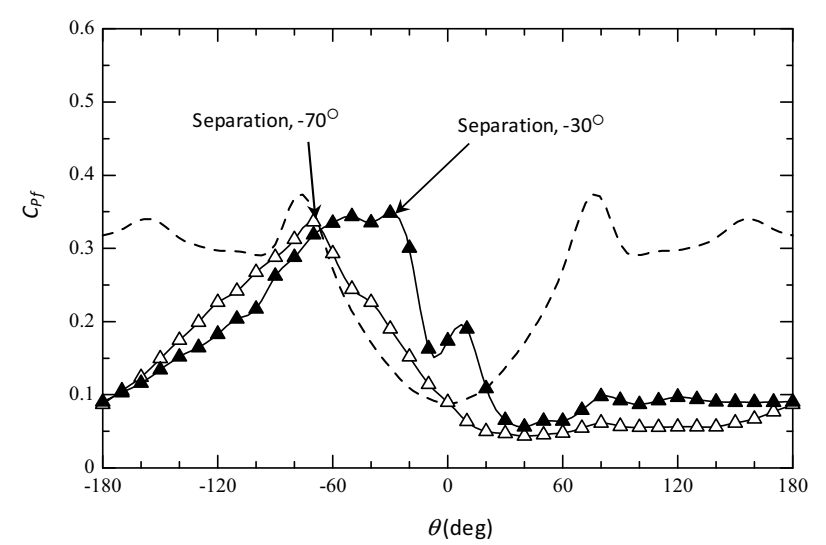

Figure 4. Fluctuating pressure coefficient $C_{P f}$ on cylinder B at $T / D=1.0, \alpha=10^{\circ}$ (downstream cylinder). $\triangle$, mode $1 ;$ mode 2 ; ---, single cylinder [6].

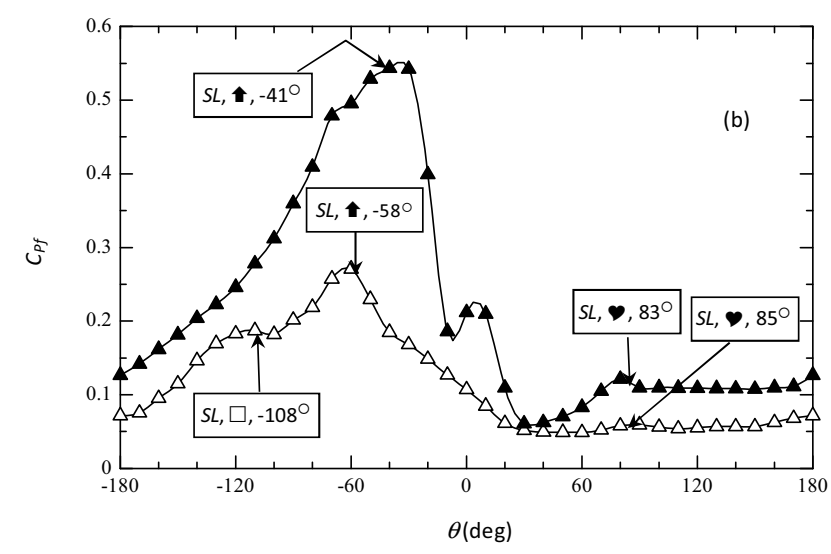

Figure 5. Fluctuating pressure coefficient $C_{P f}$ distributions on cylinder $\mathrm{B}$ at $T / D=1.0 . \triangle, \alpha=8^{\circ} ; \boldsymbol{\Delta}, \alpha=12^{\circ}$. See figure 6 for the definitions of other symbols [6].

Here attention will be paid at the point $T / D=1.0, \alpha=$ $10^{\circ}$, where $C_{L}$ is most negative and a bistable nature of flow was observed. In order to elucidate the two modes of the flow responsible for the bistability, fluctuating pressure coefficient, $C_{P f}$, on the surface of the cylinder was calculated for the two modes by employing a conditional sampling technique on digitally stored composite signals. $C_{P f}$ distributions for the two modes of the flow pattern at $T / D=1.0$ are shown in figure 4 , indicating that the outer shear layer separates from the cylinder $B$ (downstream) at the same position, irrespective of the flow modes; however, the inner shear layer separates at $\theta=-70^{\circ}$ and $-30^{\circ}$ for modes 1 and 2 , respectively. That is, the inner shear layer sweeps on the cylinder surface for a longer peripheral length for mode 1 , thus creating a higher suction pressure before separation.
Mode 1 exhibits a longer pressure recovery region on the inside surface, indicating the occurrence of the inner shear layer reattachment, which is confirmed by the surface oil-flow visualization pattern. It was found that as the staggered angle of the cylinders was slightly increased or decreased, say at $\alpha=8^{\circ}$, mode 1 only was observed, and at $\alpha=12^{\circ}$, mode 2 only was observed. The $C_{P f}$ distributions at $\alpha=8^{\circ}$ and $12^{\circ}(T / D=1.0)$ are shown in figure 5. The trends of the pressure distributions for $\alpha$ $=8^{\circ}$ and $12^{\circ}$ bear resemblance with those of the modes 1 and 2 (figure 4), respectively. Now a summary sketch of the flow pattern based on the $C_{P f}$ distributions and surface oil-flow patterns can be presented in figure 6 .

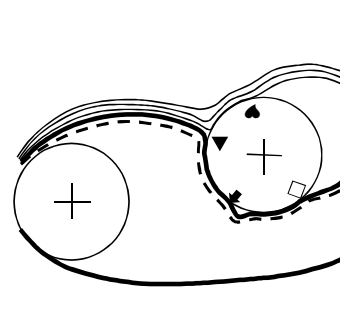

(a)

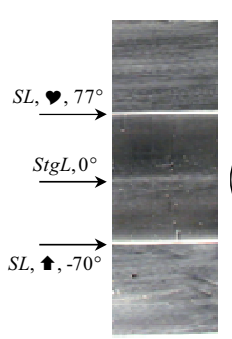

(e)

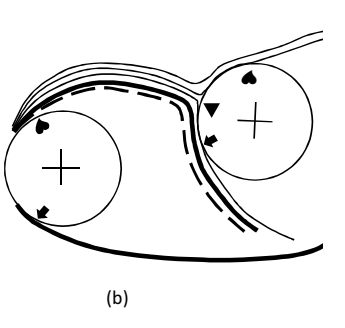

(b)

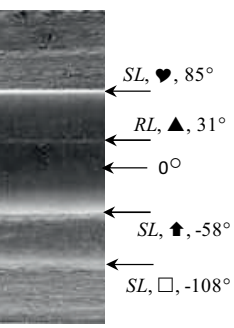

(c)

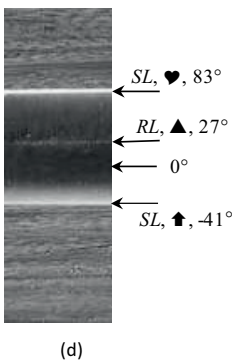

(d)
Figure 6. Flow structure sketches and surface oil-flow patterns: (a) mode 1, (b) mode 2. (c) Oil-flow pattern on cylinder B at $\alpha$ $=8^{\circ}$ and $T / D=1.0$. (d) Oil-flow pattern on cylinder B at $\alpha=$ $12^{\circ}$ and $T / D=1.0$. (e) Surface oil-flow pattern on cylinder A at $\alpha=12^{\circ}$ and $T / D=1.0 . \operatorname{Stg} L:$ stagnation line; $S L:$ separation line; $R L$ : reattachment line.

In mode 1 , as evident in surface oil-flow pattern ( $\alpha=$ $8^{\circ}$, figure $6(\mathrm{a}, \mathrm{c})$ ), the inner shear layer separating from the upstream cylinder reattaches onto the downstream cylinder at $\theta=31^{\circ}$, and the gap shear layer consisting of the high velocity middle slice and the highly turbulent inner slice sweeps on the inner surface and separates at $\theta$ $=-58^{\circ}$, followed by a turbulent reattachment at $\theta=-65^{\circ}$. The reattached shear layer finally separates at $\theta=-108^{\circ}$.

In mode $2\left(\alpha=12^{\circ}\right)$, the inner shear layer from the upstream cylinder reattaches onto the downstream cylinder at $\theta=27^{\circ}$, and the gap shear layer (the inner shear layer around the downstream cylinder) consisting 
of outer slice, high velocity middle slice and highly turbulent inner slice, passes through the gap and separates at $\theta=-41^{\circ}$. Interestingly, the separation position inferred from surface oil-flow patterns coincides with the position of the peak in the $C_{P f}$ distributions, i.e., a peak in $C_{P f}$ distribution forms at the vicinity of the separation point of a shear layer. It can be mentioned that, as the gap shear layer of mode 1 is more turbulent than that of mode 2, the gap shear layer is induced to reattach.

\section{Multistable flow detection using wavelet}

Wavelet analysis can be used to examine the intermittent appearance of two flow modes. For a given onedimensional function $u(t)$, the wavelet transform can be defined as

$$
W(s, b)=p(s) \int u(t) \Psi^{*}\left(\frac{t-b}{s}\right) \mathrm{d} t,
$$

where $W(s, b)$ is the wavelet coefficient, the superscript "*" denotes the complex conjugate, $b$ is the translation parameter, $s$ is the scale parameter. The function $\psi(t)$ is called a analyzing wavelet or mother wavelet. A wellknown function such as the Morlet function or the Mexican hat function is often used as the mother wavelet. The Mexican hat function has a better time resolution property but a poorer frequency resolution property. The Morlet function, however, has a better frequency resolution property. A real-valued wavelet function such as the Mexican hat function can isolate peaks or discontinuities in terms of frequency and time [8]. On the other hand, a complex-valued wavelet function such as the Morlet wavelet can trace out information on both amplitude and phase and is better adapted for capturing oscillatory behavior. In the present study, the Morlet function was used as a mother wavelet. This function is expressed as

$$
\Psi(\eta)=\mathrm{e}^{\mathrm{i} \omega_{0} \eta} \mathrm{e}^{-\eta^{2} / 2},
$$

where $\omega_{0}$ is the wave number in the Morlet wavelet. The bigger the wave number, $\omega_{0}$, the broader the wavelet power in time space. A narrower (in time) wavelet function has a good time resolution property but a poor frequency resolution property, while a broad wavelet function has a poor time resolution but good frequency resolution [9]. The Morlet wavelet with $\omega_{0}=6$ is marginally admissible. For the Morlet wavelet with wave number, $\omega_{0}$, the relation between scales, $s$, and the Fourier frequency is

$$
f=\frac{\omega_{0}+\sqrt{2+\omega_{0}^{2}}}{4 \pi s} .
$$

In equation (2), $p(s)$ is a weighting function that can be chosen as $s^{-1}, s^{-0.5}, s^{0.5}$ or $s^{0}$ depending on what we want to extract from the signal. The weighting function $p(s)=s^{-0.5}$ provides the same energy at each scale. However, a more negative power of $s$, such as $s^{-1}$, provides energy magnification at higher frequencies (lower scale), and nonnegative powers of $s$, such as $s^{0}$ or $s^{0.5}$, provide energy magnification at lower frequencies (higher scale).
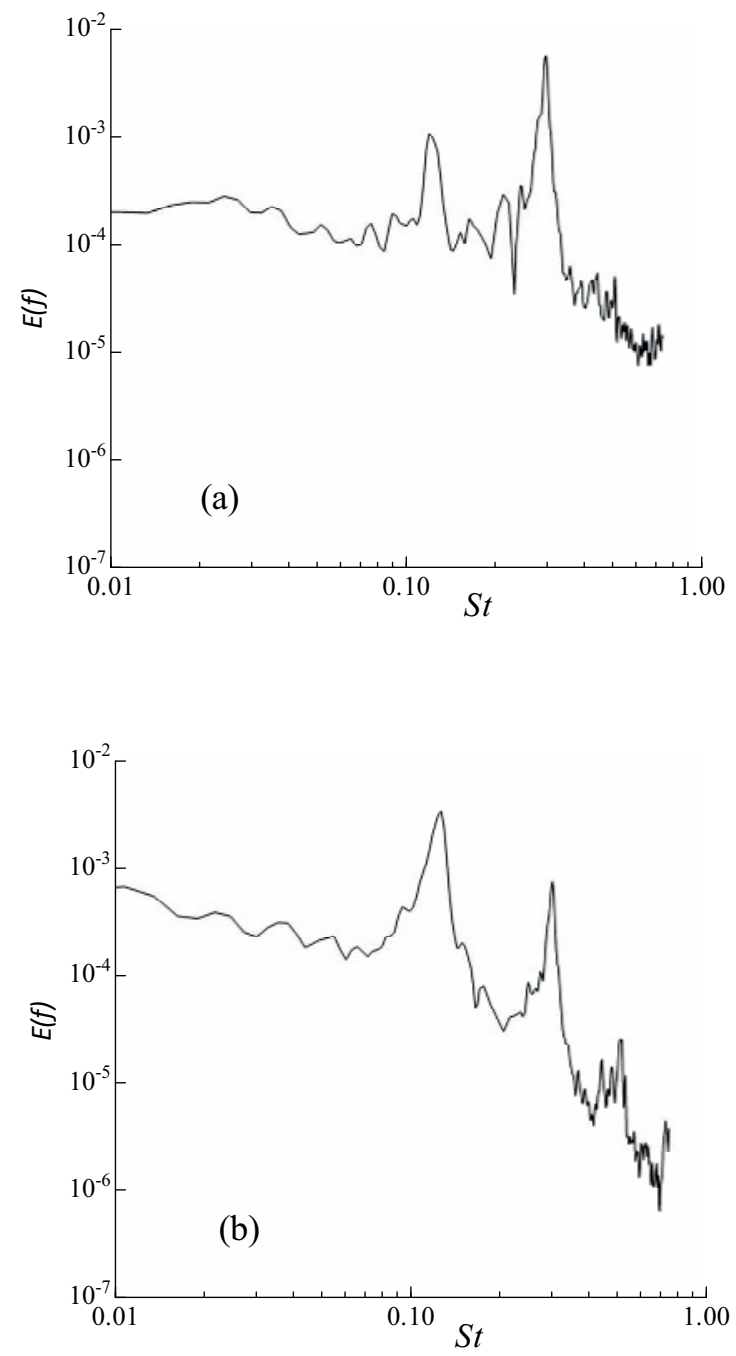

Figure 7. Power spectral density functions of fluctuating pressures on the surfaces of the cylinders at $\alpha=25^{\circ}, T / D=1.4$. (a) Cylinder A $\left(\theta=90^{\circ}\right)$. (b) Cylinder $B\left(\theta=90^{\circ}\right)$.

Two peaks in Fourier power spectra of fluctuating pressure signals on either side of each cylinder were found for $T / D=1.1 \sim 1.8$. Typical Fourier power spectra shown in figure 7 show two $S t$ for each of the cylinders. The level of energy at the higher $S t$ of the upstream cylinder and at the lower $S t$ of the downstream cylinder is higher. Now it is not clear how the two St appears, simultaneously or separately in time space? Timefrequency analysis of the signals could be an effective 
way to clearly illustrate their presences. Figure 8 shows wavelet scalograms of fluctuating pressure signals for the upstream and downstream cylinders at $T / D=1.40$. The pressure signals are at $\theta=90^{\circ}$ for both the cylinders, i.e., they capture information on the characteristics of the outer shear layers only. The wavelet scalograms evidence that the upstream and downstream cylinders shed vortices with a high intensity of energy when the frequency of vortex shedding is high and low, respectively. This is prevalent but the outer shear layer of the upstream cylinder frequently sheds vortices at the lower frequency of the downstream cylinder and the outer shear layer of the downstream cylinder frequently sheds vortices at the higher frequency of the upstream cylinder. In other words, the vortex shedding of the upstream cylinder frequently locks-in at the lower frequency of the downstream cylinder and that of the downstream cylinder also frequently locks-in at the higher frequency of the upstream cylinder. However, the length of the lock-in time for the upstream cylinder to shed vortices at the lower frequency is comparatively shorter and that for the downstream cylinder to shed vortices with the higher frequency is considerably longer. Mutual interactions of the four shear layers cause such lock-in phenomena. The lock-in of the frequency of the downstream cylinder with that of the upstream one can be supposed to be contributed from the tandem effect; as in tandem arrangement, the downstream cylinder is forced to shed vortices at the frequency of the upstream cylinder. On the other hand, intermittent lock-in of the upstream cylinder shedding with the downstream can be explained as the flow pattern that appears for $T / D<1.1$, a single bluff-body flow with regard to vortex-shedding frequency, the lower $S t$ for both the cylinders, continues its appearance intermittently up to $T / D=1.8$. Hence there are three stable flow patterns at $T / D=1.1 \sim 1.8$ with regard to $S t$ : (i) the flow with a higher $S t$ for the upstream cylinder and a lower $S t$ for the downstream cylinder, for example the flow at the neighborhood of $t=$ $0.7 \mathrm{sec}$, (ii) the flow with a higher $S t$ for both the cylinders: lock-in of the downstream cylinder wake to the upstream one, for example the flow at the neighborhood of $t=0.1$ and $1.4 \mathrm{sec}$, and (iii) the flow with a lower $S t$ for both the cylinders: lock-in of the upstream cylinder wake to the downstream one, for example the flow at the neighborhood of $t=0.6,0.9$ and $1.15 \mathrm{sec}$. It is also seen in the wavelet maps that when the downstream cylinder locks-in to shed vortices at the higher frequency of upstream cylinder, the energy level at the higher frequency of the upstream cylinder becomes greater, implying relatively stronger vortices are shed from the upstream cylinder when both the cylinder shed vortices with the higher frequency.

\section{Conclusions}

(1) The $C_{L}$ for $T / D<0.5$ varies greatly with change in $\alpha$ from $0^{\circ}$ to $360^{\circ}$. The downstream cylinder experiences a highly negative $C_{L}$ at $\alpha=10^{\circ}, T / D=$ 0.9 and the upstream cylinder at $\alpha=155^{\circ}, T / D=0.3$. The highly negative $C_{L}$ on the upstream cylinder at $\alpha$ $=155^{\circ}, T / D=0.3$ is mainly due to a greater circulation of the gap flow on the inner and rear surfaces of the upstream cylinder. The upstream cylinder experiences highly positive $C_{L}$ at $\left(\alpha=135^{\circ}\right.$, $T / D=0.1),\left(\alpha=120^{\circ}, T / D=0.2\right)$ and $\left(\alpha=105^{\circ}, T / D\right.$ $=0.2$ ). These highly positive $C_{L}$ are due to shift of stagnation point toward the inside surface of the upstream cylinder and retardation of flow on the inside surface of the upstream cylinder by the front surface of the downstream cylinder. There exist three island-like regimes where the value of $C_{L f}$ are extensively high, about 1.58 times the single cylinder values.

(2) When there is a bistable flow, conditional sampling technique can be employed to get individual pressure distributions corresponding to the individual modes. Flow separation and reattachment points obtained from surface oil-flow image and fluctuating pressure distribution agree well each other.

(3) The multistable flow patterns and the lock-in phenomenon are elucidated from the results of wavelet analysis of fluctuating pressures simultaneously stored from the surfaces of the two cylinders. Wavelet analysis is a very useful tool for analyzing multistable flow, lock-in phenomenon and mutual change of two flows in time domain.

\section{Acknowledgements}

The author wishes to acknowledge support given to him from Shenzhen Government through grant CB24405004 and from China Govt through '1000-young-talentprogram'. The author is also grateful for Professor Hiroshi Sakamoto, Kitami Institute of Technology, for his support to conduct some experiments his laboratory.

\section{References}

1. D. Sumner, S.J. Price, M.P. Paidoussis, J. Fluid Mech. 411 (2000).

2. Z. Gu, T. Sun, J. Wind Eng. Indust. Aerodyn. 80 (1999).

3. A. Bokaian, F. Geoola, J. Fluid Mech. 146 (1984).

4. S.J. Price, M.P. Paidoussis, J. Wind Eng. Indust. 
Aerodyn. 17 (1984).

5. M.M. Alam, M. Moriya, H. Sakamoto, J. Fluids Struct. 18 (2003).

6. M.M. Alam, H. Sakamoto, Y. Zhou, J. Fluids Struct. 21 (2005).

7. R.M.C. So, S.D. Savkar, J. Fluid Mech. 105 (1981).
8. J. Lewalle, AIAA J. 94 (1994).

9. C. Torrence, G. Compo, Bull. American Meteorological Soc. 79 (1998).

10. M.M. Alam, J.P. Meyer, Phy. Rev. E. 84 (2011).

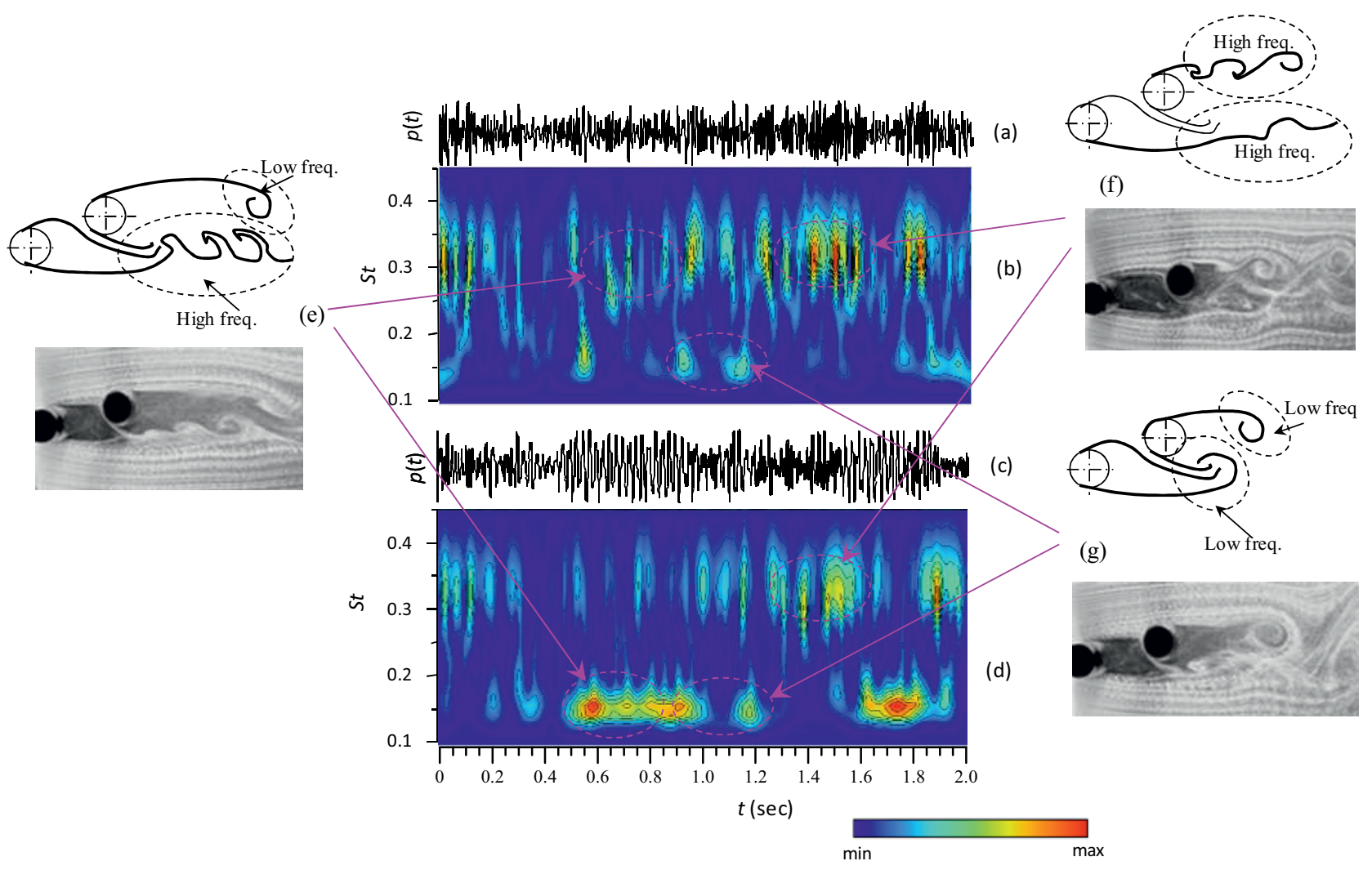

Figure 8. (b, d) Wavelet scalograms of pressure signals from (a) cylinder $A$ at $\theta=90^{\circ}$ and (c) cylinder $B$ at $\theta=90^{\circ}$, respectively. (e-g) Flow visualization photographs and sketches corresponding to $S t$ in wavelet scalogram. Signals (a) and (c) stored simultaneously. $\alpha=25^{\circ}, T / D=1.4$. 